\title{
A Seismic Blind Deconvolution Algorithm Based on Bayesian Compressive Sensing
}

\author{
Yanqin $\mathrm{Li}^{1,2}$ and Guoshan Zhang \\ ${ }^{1}$ School of Electrical Engineering \& Automation, Tianjin University, Tianjin 300072, China \\ ${ }^{2}$ Department of Disaster Prevention Equipment, Institute of Disaster Prevention, Beijing 101601, China \\ Correspondence should be addressed to Guoshan Zhang; zhanggs@tju.edu.cn
}

Received 19 March 2015; Accepted 20 April 2015

Academic Editor: Wanquan Liu

Copyright (c) $2015 \mathrm{Y}$. Li and G. Zhang. This is an open access article distributed under the Creative Commons Attribution License, which permits unrestricted use, distribution, and reproduction in any medium, provided the original work is properly cited.

\begin{abstract}
Compressive sensing in seismic signal processing is a construction of the unknown reflectivity sequence from the incoherent measurements of the seismic records. Blind seismic deconvolution is the recovery of reflectivity sequence from the seismic records, when the seismic wavelet is unknown. In this paper, a seismic blind deconvolution algorithm based on Bayesian compressive sensing is proposed. The proposed algorithm combines compressive sensing and blind seismic deconvolution to get the reflectivity sequence and the unknown seismic wavelet through the compressive sensing measurements of the seismic records. Hierarchical Bayesian model and optimization method are used to estimate the unknown reflectivity sequence, the seismic wavelet, and the unknown parameters (hyperparameters). The estimated result by the proposed algorithm shows the better agreement with the real value on both simulation and field-data experiments.
\end{abstract}

\section{Introduction}

Digital seismic signal receiving devices have been developed for many years. In traditional seismic signal receiving system, the accurate signal is obtained by Nyquist sampling theorem which claims that the seismic signal sampling rate should be at least twice the seismic signal's highest frequency. Measurement reduction, such as compressive sensing (CS), can make the seismic record devices be simpler, smaller, and cheaper. CS is a signal processing method which can reconstruct the original signal from a small number of random incoherent liner measurements $[1,2]$. CS uses the property of the signal's sparseness or compressibility in some domain, which achieves the entire signal from relatively few measurements [3]. CS has become very popular in recent years because it can reduce the number of data transmissions and give potential aid in many practical applications $[4,5]$.

The CS problem is to compress data from original data while the inverse CS problem is to reconstruct original data from the compressed data. There exist lots of effective methods to solve the CS problem. Some of these methods are based on energy limit $[6,7]$ and the others are based on the Bayesian framework $[8,9]$. Bayesian compressive sensing gives a Bayesian framework for solving the inverse problem of compressive sensing (CS). The basic Bayesian CS algorithm exploits the relevance vector machine and then it marginalizes the noise variance with improved robustness $[10,11]$.

Deconvolution, as a seismic data processing technique, has been widely used to yield reflectivity sequence from the seismic records [12-15]. The Bernoulli-Gaussian model introduced in [16], as the reflectivity sequence model, is popular. It is very intuitive and its statistical properties can easily apply in Bayesian methods [17-19] such as Gibbs sampling and MCMC (Markov Chain Monte Carlo) techniques. In statistical models, an expectation maximization (EM) algorithm is an iterative method for finding maximum likelihood (ML) or maximum a posteriori (MAP) estimates of parameters, where the model depends on unobserved latent variables [20]. So, EM algorithm is usually used to estimate the unknown parameters (the wavelet, parameters of the BG model and additive noise variance) in blind seismic deconvolution. The EM algorithm iteration alternates between performing an expectation (E) step and a maximization (M) step. 
E step creates a function for the expectation of the loglikelihood while $\mathrm{M}$ step computes parameters maximizing the expected log-likelihood found on the E step. These parameter-estimates are then used to determine the distribution of the latent variables in the next E step.

The Bayesian method is model-based method which is applied to estimate unknown parameters using the prior information. So it can handle observation noise and missing data problems [21]. Moreover, sparsity, as the important characteristic of a signal, has been exploited in many application areas, such as seismic deconvolution; see [22-24]. It could be an alternative method for the deconvolution of sparse signals that model a sparse signal with a Gaussian random variable whose variance is an inverse-Gamma random variable [2529]. It exploits variational Bayesian techniques for a closedform approximate posterior inference [30].

In this paper, the reflectivity sequence and the seismic wavelet are recovered through CS measurement of the seismic records. Bayesian framework is used in CS problem in seismic signal processing. Blind seismic deconvolution and optimization method are combined to estimate the unknown reflectivity sequence, the seismic wavelet, and the hyperparameters. The organization of this paper is as follows. In Section 2, the Bayesian model with CS measurement of the blind seismic restoration problem is given. In Section 3, the observation model and the prior model on the signals coefficients are presented. In Section 4, the Bayesian inference is presented. Optimization method is used to get the solution of the unknown reflectivity sequence and the parameters. In Section 5, comparison between ML and Bayesian deconvolution with CS measurement is given based on the both simulation and field data experiments. In the last section, we draw the conclusions.

\section{Problem Formulation}

Seismic record is the convolution with the seismic wavelet and the reflectivity sequence. A standard seismic record image model is given in a matrix-vector form by

$$
\mathbf{y}=\mathbf{S r}+\mathbf{n},
$$

where $\mathbf{y}(n)=[y(1), \ldots y(M)]^{\mathrm{T}}$ denotes a seismic observation vector, $\mathbf{r}(n)=[r(1), \ldots, r(N)]^{\mathrm{T}}$ denotes an unknown seismic reflectivity sequence vector, and $\mathbf{n}(n)=[n(1), \ldots, n(M)]^{\mathrm{T}}$ is an additive white Gaussian noise vector, respectively. $\mathbf{S}$, which represents seismic wavelet, is a $M \times N$ block Toeplitz matrix obtained by a block-circulant matrix. The seismic wavelet and reflectivity vector are both unknown, so the deconvolution is a blind problem.

Considering the sparsity of the seismic reflectivity vector, $\mathbf{r}$ is compressible by a linear wavelet basis $\Psi$; that is, $\mathbf{r}=\Psi \mathbf{w}$, where $\mathbf{w}$ is a $N \times 1$ sparse signal. Reference [18] proposed sparse schemes that capture the convolution structure of pulse streams by defining (2) which resorts to a CS matrix $\Phi$ to complete $M \ll N$ measurements.

Equation (1) can be rewritten in the following form:

$$
\mathbf{y}=\Phi \mathbf{w}+\mathbf{n},
$$

where $\Phi=\mathbf{S} \Psi$. Based on the sparsity of $\mathbf{w}$, the $l_{1}$ norm of $\mathbf{w}$ is used in the inverse problem. The estimation of the original signal can be obtained by solving the following optimization problem [31]:

$$
\widehat{\mathbf{w}}=\underset{\mathbf{w}}{\arg \min }\left\{\|\mathbf{y}-\Phi \mathbf{w}\|_{2}^{2}+\tau\|\mathbf{w}\|_{1}\right\},
$$

where $\|\cdot\|_{1}$ represents the $l_{1}$ norm.

\section{Bayesian Modeling}

The prior distribution of unknown signal $\mathbf{w}$ is assumed to be $p(\mathbf{w} \mid \alpha)$. The observation $\mathbf{y}$ is the random process with distribution $p(\mathbf{y} \mid \mathbf{w}, \beta)$, where $\beta^{-1}$ denotes the unknown noise variance. These seismic record and reflectivity depend on the unknown hyperparameters $\alpha$ and $\beta$. Following the Bayesian framework of the CS inverse problem, the following joint posterior distribution can be expressed by

$$
p(\mathbf{w}, \alpha, \beta, \mathbf{y})=p(\mathbf{y} \mid \mathbf{w}, \beta) p(\mathbf{w} \mid \alpha) p(\alpha) p(\beta) .
$$

In Bayesian estimation, all unknown parameters are assumed to be random variables with specific distribution. Our goal is to estimate the parameters $\mathbf{S}, \mathbf{w}, \alpha$, and $\beta$ from $\mathbf{y}$. The parameter $\mathbf{S}$ can be estimated by the Stochastic expectation maximization algorithm of Rosec et al. [32]. The following gives how to get the other parameters.

3.1. Seismic Observation Modeling. By assuming that the seismic data noise in (2) is Gaussian distribution with zero mean and variance equal to $\beta^{-1}$, the conditional distribution of the seismic record is

$$
p(\mathbf{y} \mid \mathbf{w}, \beta)=N\left(\mathbf{y} \mid \Phi \mathbf{w}, \beta^{-1}\right)
$$

with a Gamma prior distribution on $\beta$ as the following:

$$
p\left(\beta \mid a^{\beta}, b^{\beta}\right)=\Gamma\left(\beta \mid a^{\beta}, b^{\beta}\right) .
$$

The Gamma distribution for the hyperpriors $\beta$ is defined as

$$
p(x)=G\left(x \mid a^{x}, b^{x}\right)=\frac{\left(b^{x}\right)^{a^{x}}}{\Gamma\left(a^{x}\right)}(x)^{a^{x}-1} \exp \left(-b^{x} x\right),
$$

where $x>0$ represents hyperparameter, $a^{x}$ is the scale parameter, and $b^{x}$ is the shape parameter. These parameters are also assumed to be random variables. We will show how they are calculated in the following subsection. The Gamma distribution has the following mean and variance:

$$
\begin{gathered}
E[x]=\frac{a^{x}}{b^{x}} \\
\operatorname{Var}[x]=\frac{a^{x}}{\left(b^{x}\right)^{2}} .
\end{gathered}
$$


3.2. Signal Modeling. In [33], as the reflectivity sequence also has sparsity, w can use the following prior model:

$$
p(\mathbf{w} \mid \boldsymbol{\alpha})=\prod_{i=1}^{N} N\left(w_{i} \mid 0, \alpha_{i}\right),
$$

where $\boldsymbol{\alpha}=\left(\alpha_{1}, \alpha_{2}, \ldots, \alpha_{N}\right)$. Then, hyperprior of the hyperparameter $\alpha_{i}$ is as the following:

$$
\begin{aligned}
& p\left(\alpha_{i} \mid \lambda\right)=\Gamma\left(\alpha_{i} \mid 1, \frac{\lambda}{2}\right)=\frac{\lambda}{2} \exp \left(-\frac{\lambda \alpha_{i}}{2}\right), \\
& \alpha_{i} \geq 0, \lambda \geq 0 .
\end{aligned}
$$

Based on (9) and (10), we have the following equation:

$$
\begin{aligned}
p(\mathbf{w} \mid \lambda) & =\int p(\mathbf{w} \mid \boldsymbol{\alpha}) p(\boldsymbol{\alpha} \mid \lambda) d \boldsymbol{\alpha} \\
& =\prod_{i} \int p\left(w_{i} \mid \alpha_{i}\right) p\left(\alpha_{i} \mid \lambda\right) d \alpha_{i} \\
& =\frac{\lambda^{N / 2}}{2^{N}} \exp \left(-\lambda^{1 / 2} \sum_{i}\left|w_{i}\right|\right) .
\end{aligned}
$$

At last, hyperparameter $\lambda$ is modeled as the following Gamma hyperprior:

$$
p(\lambda \mid \nu)=\Gamma\left(\lambda \mid \frac{\nu}{2}, \frac{v}{2}\right)
$$

The modeling has three stage forms. The first stage is the prior distribution about $\mathbf{w}$ in (9). The second stage is the prior distribution about $\alpha$ in (10). Finally, (12) is used to calculate $\lambda$. So we can first sample a $\Gamma(\nu / 2, \nu / 2)$ from the prior distribution on $\mathbf{w}$ distribution to get $\lambda$ and then sample a $\Gamma(1, \lambda / 2)$ distribution $N$ times to get $\lambda_{i}, i=1, \ldots, N$, and at last sample a $N\left(0, \alpha_{i}\right)$ distribution to get $w_{i}$.

Based on the above, the joint distribution can be given as the following:

$$
\begin{aligned}
& p(\mathbf{w}, \boldsymbol{\alpha}, \lambda, \beta, \mathbf{y}) \\
& \quad=p(\mathbf{y} \mid \mathbf{w}, \beta) p(\beta) p(\mathbf{w} \mid \boldsymbol{\alpha}) p(\boldsymbol{\alpha} \mid \lambda) p(\lambda),
\end{aligned}
$$

where $p(\mathbf{y} \mid \mathbf{w}, \beta), p(\beta), p(\mathbf{w} \mid \boldsymbol{\alpha}), p(\boldsymbol{\alpha} \mid \lambda)$, and $p(\lambda)$ are defined in (5), (6), (9), (10), and (12), respectively.

The seismic record variables of the proposed model are $\left\{y_{i}\right\}$. The parameter is $\left\{w_{i}\right\}$ while the hyperparameters are $\left\{\alpha_{i}\right\}$ and $\left\{\beta_{i}\right\}$. The parameter of the hyperparameters model is $\lambda$. The dependencies among the random variables that define the proposed Bayesian compressive sensing model are shown in Figure 1.

Given the initial estimates $q^{1}(\mathbf{w}), q^{1}(\boldsymbol{\alpha}), q^{1}(\beta)$, and $q^{1}(\lambda)$, we now proceed to explicitly calculate the distributions $q^{k}(\mathbf{w}), q^{k+1}(\boldsymbol{\alpha}), q^{k+1}(\beta)$, and $q^{k+1}(\lambda)(k=1,2 \ldots)$ until a convergence criterion is met in the above algorithm. Then, the means above can be used to recalculate the distributions of reflectivity in the above algorithm. The performance of the algorithm heavily depends on the level of the noise and the initial distributions used in the iterative algorithm.

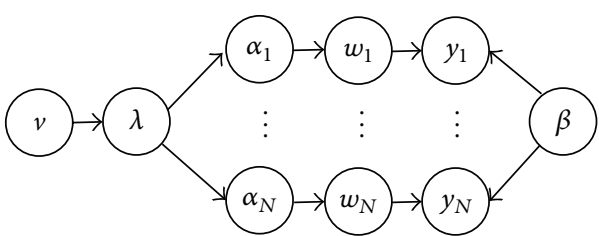

FIgURE 1: Graphical model describing the data-generation process for the blind deconvolution problem.

\section{Bayesian Inference}

The following Bayesian inference posterior distribution is well known; that is,

$$
p(\mathbf{w}, \boldsymbol{\alpha}, \lambda, \beta \mid \mathbf{y})=\frac{p(\mathbf{w}, \boldsymbol{\alpha}, \lambda, \beta, \mathbf{y})}{p(\mathbf{y})} .
$$

However, the posterior $p(\mathbf{w}, \boldsymbol{\alpha}, \lambda, \beta \quad \mathbf{y})$ cannot be obtained just because

$$
p(\mathbf{y})=\iiint \int p(\mathbf{w}, \boldsymbol{\alpha}, \lambda, \beta, \mathbf{y}) d \mathbf{w} d \boldsymbol{\alpha} d \lambda d \beta
$$

and $p(\mathbf{y})$ is not easy to be calculated directly. So, the proper method should be given to perform the estimation. Evidence procedure is exploited to complete Bayesian inference in this paper.

Bayesian inference is derived by the evidence procedure which is based on the following decomposition:

$$
p(\mathbf{w}, \boldsymbol{\alpha}, \lambda, \beta \mid \mathbf{y})=p(\mathbf{w} \mid \mathbf{y}, \boldsymbol{\alpha}, \beta, \lambda) p(\boldsymbol{\alpha}, \beta, \lambda \mid \mathbf{y})
$$

For $p(\mathbf{w} \mid \mathbf{y}, \boldsymbol{\alpha}, \beta, \lambda) \propto p(\mathbf{w}, \mathbf{y}, \boldsymbol{\alpha}, \beta, \lambda)$, the distribution of $p(\mathbf{w} \mid \mathbf{y}, \boldsymbol{\alpha}, \beta, \lambda)$ is assumed to be a multivariate Gaussian distribution $N(\mathbf{w} \mid \mu, \Sigma)$. The mean $\mu$ and the variance $\Sigma$ are

$$
\begin{aligned}
& \mu=\Sigma \beta \Phi^{\mathrm{T}} \mathbf{y} \\
& \Sigma=\left[\beta \Phi^{\mathrm{T}} \Phi+\Lambda\right],
\end{aligned}
$$

with

$$
\Lambda=\operatorname{diag}\left(\frac{1}{\alpha_{i}}\right)
$$

Since the distribution $p(\boldsymbol{\alpha}, \beta, \lambda \mid \mathbf{y})=p(\mathbf{y}, \boldsymbol{\alpha}, \beta, \lambda) / p(\mathbf{y})$ is proportional to $p(\mathbf{y}, \boldsymbol{\alpha}, \beta, \lambda)$, the hyperparameters can be obtained by maximizing the joint distribution $p(\mathbf{y}, \boldsymbol{\alpha}, \beta, \lambda)$. So we get

$$
\begin{aligned}
& p(\mathbf{y}, \boldsymbol{\alpha}, \beta, \lambda) \\
& \quad=\int p(\mathbf{y} \mid \mathbf{w}, \beta) p(\mathbf{w} \mid \boldsymbol{\alpha}) p(\boldsymbol{\alpha} \mid \lambda) p(\beta) d \mathbf{w} \\
& \quad=\left(\frac{1}{2 \pi}\right)^{N / 2}\left|\beta^{-1} \mathbf{I}+\Phi \Lambda^{-1} \Phi^{\mathrm{T}}\right|^{-1 / 2}
\end{aligned}
$$




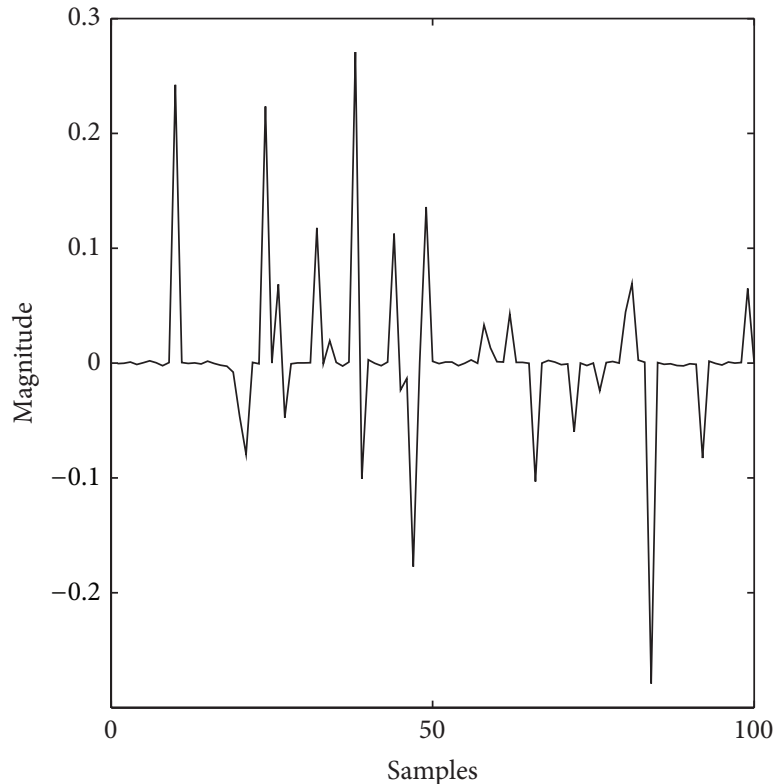

(a)

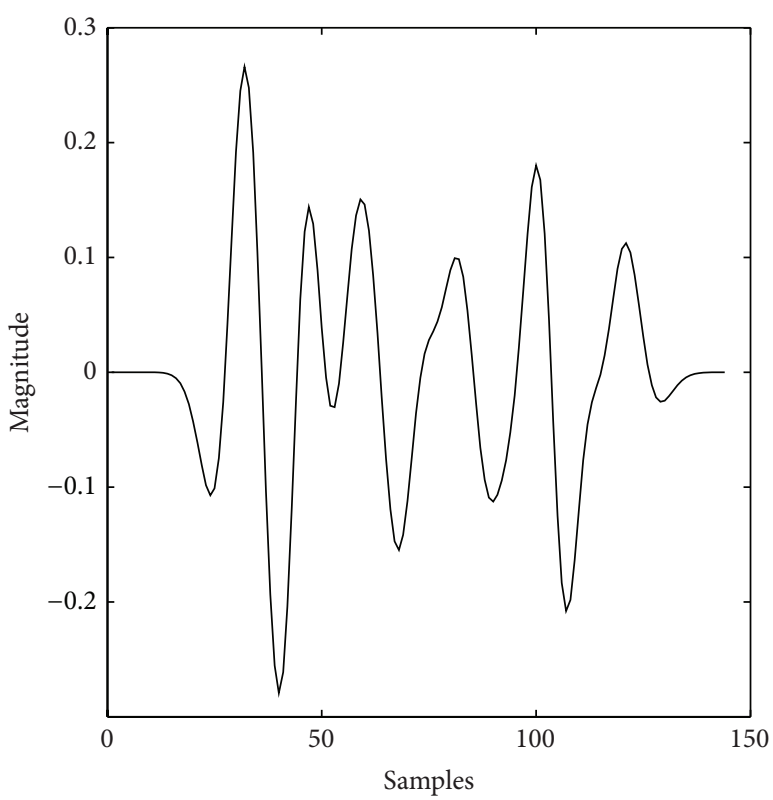

(c)

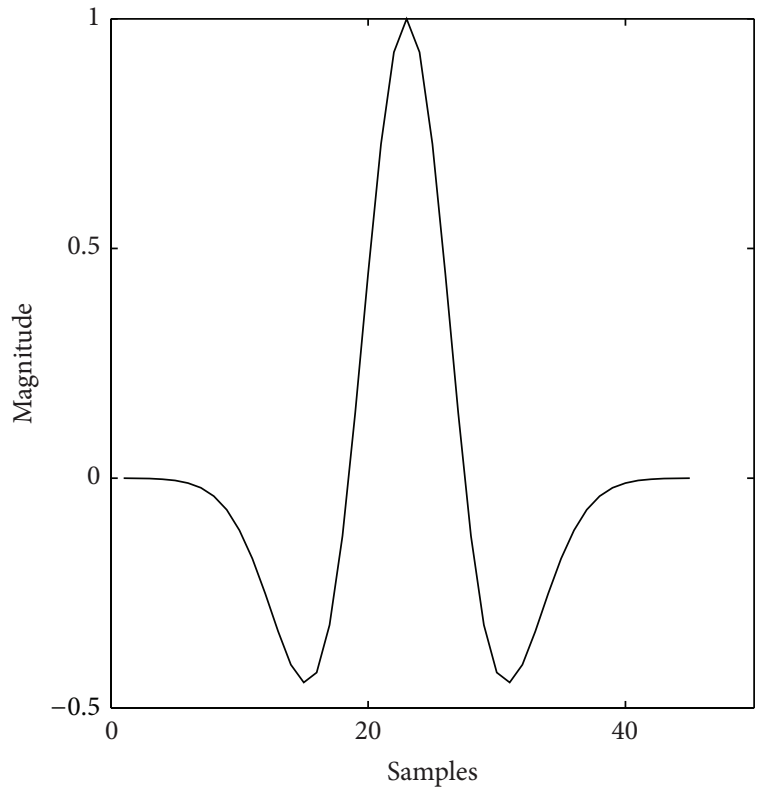

(b)

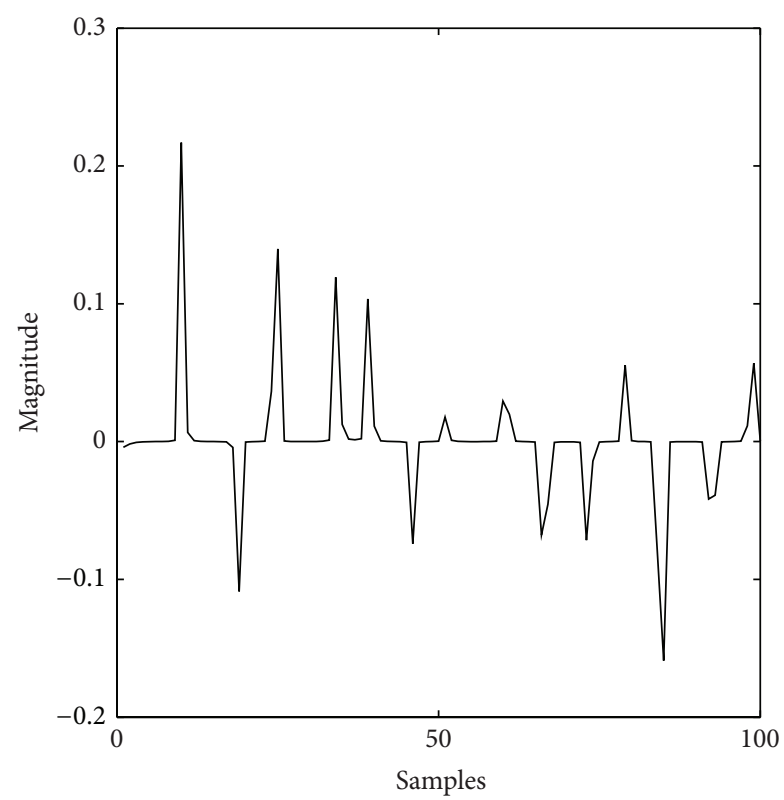

(d)

FIGURE 2: Synthetic reflectivity, wavelet, and data sets. (a) Synthetic $1 \mathrm{D}$ reflectivity sequence. (b) $1 \mathrm{D}$ seismic wavelet. (c) $1 \mathrm{D}$ seismic data (SNR = $10 \mathrm{~dB}$ ). (d) Estimated reflection coefficients (Bayesian compressive sensing).

$$
\begin{aligned}
& \cdot \exp \left[-\frac{1}{2} \mathbf{y}^{\mathrm{T}}\left(\beta^{-1} \mathbf{I}+\Phi \Lambda^{-1} \Phi^{\mathrm{T}}\right)^{-1} \mathbf{y}\right] p\left(\frac{\boldsymbol{\alpha}}{\lambda}\right) p(\lambda) \\
& \cdot p(\beta)=\left(\frac{1}{2 \pi}\right)^{N / 2}|\mathbf{C}|^{-1 / 2} \exp \left[-\frac{1}{2} \mathbf{y}^{\mathrm{T}} \mathbf{C}^{-1} \mathbf{y}\right] \\
& =p\left(\frac{\boldsymbol{\alpha}}{\lambda}\right) p(\lambda) p(\beta)
\end{aligned}
$$

where $\mathbf{I}$ is $M \times M$ identity matrix and $\mathbf{C}=\left(\beta \mathbf{I}+\Phi \Lambda^{-1} \Phi^{\mathrm{T}}\right)$.
Taking the logarithm of (19) and then maximizing the result, we can get the following function:

$$
\begin{aligned}
L= & \frac{1}{2} \log |C|-\frac{1}{2} y^{\mathrm{T}} C^{-1} y+N \log \frac{\lambda}{2}-\frac{\lambda}{2} \sum_{i} \alpha_{i} \\
& +\frac{v}{2} \log \frac{\nu}{2}-\log \Gamma\left(\frac{v}{2}\right)+\left(\frac{v}{2}-1\right) \log \lambda-\frac{v}{2} \lambda \\
& +\left(a^{\beta}-1\right) \log \beta-b^{\beta} \beta .
\end{aligned}
$$




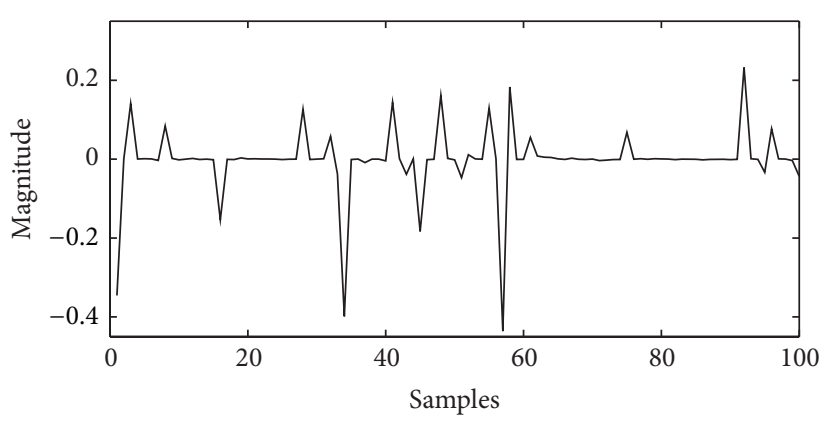

(a)

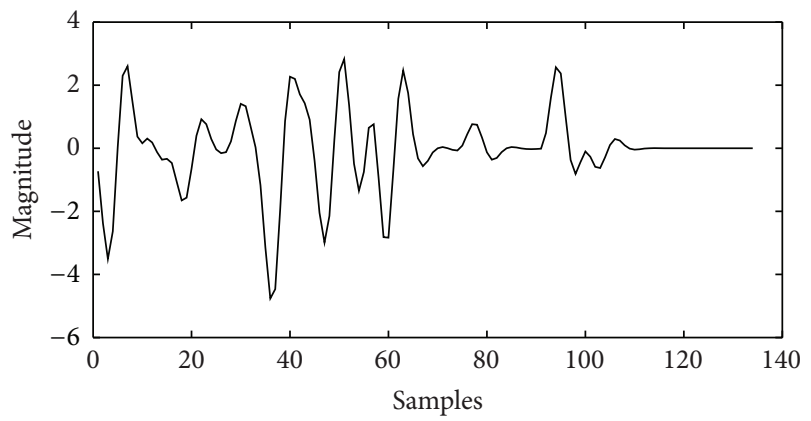

(c)

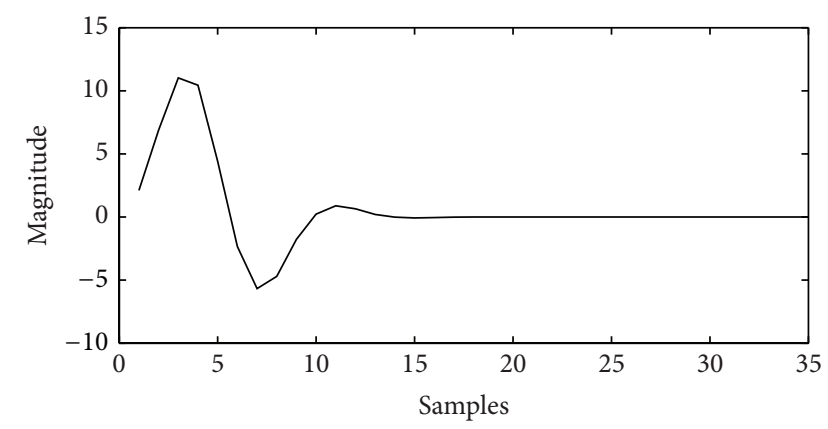

(b)

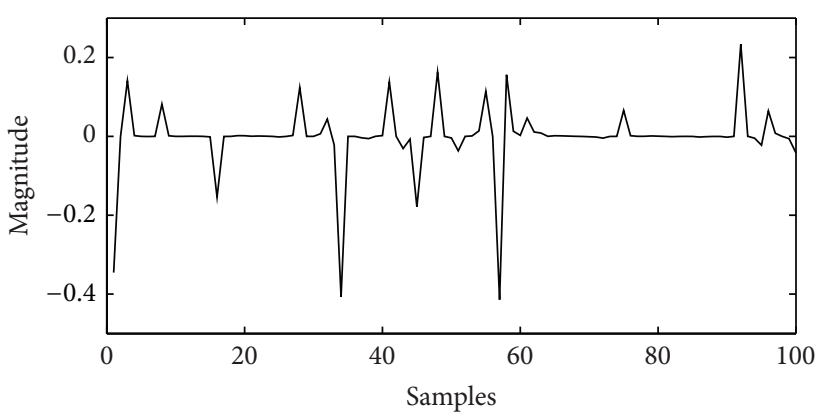

(d)

FIGURE 3: Synthetic reflectivity, wavelet, and data sets. (a) Synthetic 1D reflectivity sequence. (b) 1D seismic wavelet. (c) 1D seismic data (SNR $=20 \mathrm{~dB}$ ). (d) Estimated reflection coefficients (Bayesian compressive sensing).

In order to get the solution of the maximization problem, we give the following equations below. Consider

$$
|\mathbf{C}|=|\Lambda|^{-1}\left|\beta^{-1} \mathbf{I}\right|\left|\Lambda+\beta \Phi^{\mathrm{T}} \Phi\right|=|\Lambda|^{-1}\left|\beta^{-1} \mathbf{I}\right|\left|\Sigma^{-1}\right| .
$$

Taking the logarithm of (21),

$$
\log |C|=-\log |\Lambda|-N \log \beta-\log |\Sigma|,
$$

where

$$
\begin{aligned}
\mathbf{C}^{-1} & =\left(\beta^{-1} \mathbf{I}+\Phi \Lambda^{-1} \Phi^{\mathrm{T}}\right)^{-1} \\
& =\beta \mathbf{I}-\beta \Phi\left(\Lambda+\beta \Phi^{\mathrm{T}} \Phi\right)^{-1} \Phi^{\mathrm{T}} \beta \\
& =\beta \mathbf{I}-\beta \Phi \Sigma \Phi^{\mathrm{T}} \beta .
\end{aligned}
$$

The following function is obtained by (23) and (17):

$$
\begin{aligned}
\mathbf{y}^{\mathrm{T}} \mathbf{C}^{-1} \mathbf{y} & =\beta \mathbf{y}^{\mathrm{T}} \mathbf{y}-\beta \mathbf{y}^{\mathrm{T}} \Phi \Sigma \Phi^{\mathrm{T}} \beta \mathbf{y}=\beta \mathbf{y}^{\mathrm{T}}(\mathbf{y}-\Phi \mu) \\
& =\beta\|\mathbf{y}-\Phi \mu\|^{2}+\beta \mu^{\mathrm{T}} \Phi^{\mathrm{T}}(\mathbf{y}-\Phi \mu) \\
& =\beta\|\mathbf{y}-\Phi \mu\|^{2}+\mu^{\mathrm{T}} \Lambda \mu .
\end{aligned}
$$

Therefore, based on the above equations and differential of the equation $L$ with respect to $\alpha_{i}$, we obtain

$$
\frac{d L}{d \alpha_{i}}=\frac{1}{2}\left[-\frac{1}{\alpha_{i}}+\frac{\left\langle w_{i}^{2}\right\rangle}{\alpha_{i}^{2}}-\lambda\right],
$$

where $\left\langle w_{i}^{2}\right\rangle=\mu_{i}^{2}+\Sigma_{i j}$ and $\Sigma_{i j}$ denotes the $i$ th diagonal element of $\Sigma$. Making (25) be zero, we can obtain the solution of $\alpha_{i}$ :

$$
\alpha_{i}=-\frac{1}{2 \lambda}+\left(\frac{1}{4 \lambda^{2}}+\frac{\left\langle w_{i}^{2}\right\rangle}{\lambda}\right)
$$

Similarly, the other hyperparameters are updated in the following manner, which is given by

$$
\begin{aligned}
& \lambda=\frac{N-1+v / 2}{\sum_{i} \alpha_{i} / 2+\nu / 2} \\
& \beta=\frac{N / 2+a^{\beta}}{\left\langle\|y-\Phi w\|^{2}\right\rangle / 2+b^{\beta}} .
\end{aligned}
$$

The hyperparameter $v$ satisfies the following equation:

$$
\log \frac{v}{2}+1-\phi\left(\frac{\nu}{2}\right)+\log \lambda-\lambda=0
$$

where $\varphi(\cdot)=\ln \Gamma(x)$.

Generally speaking, the hyperparameters $\alpha, \lambda, \beta, \nu$ are iterated by (26), (27), and (28). The parameter $\mathbf{w}$ is estimated by (17). 


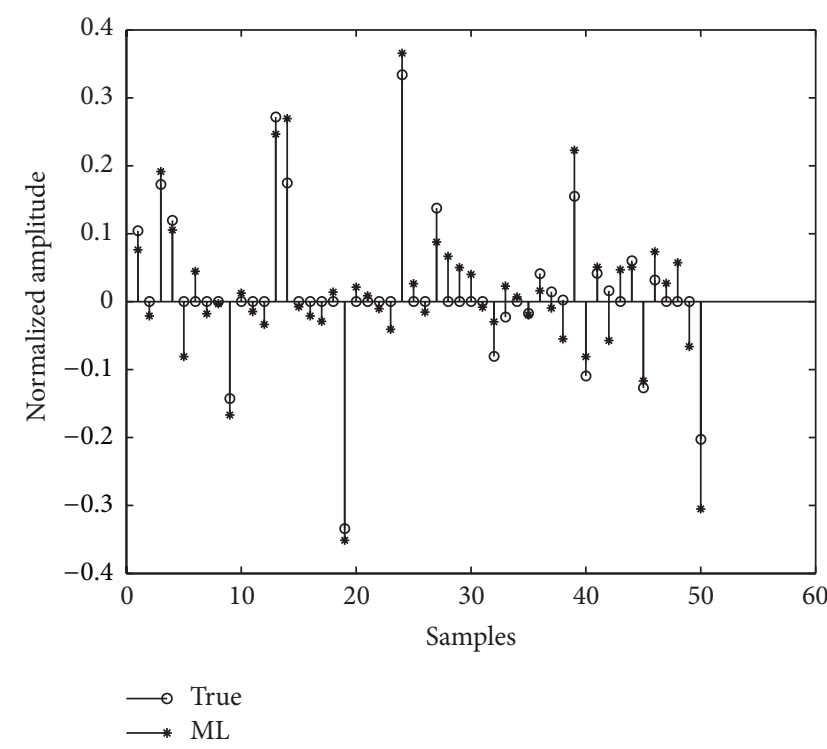

(a)

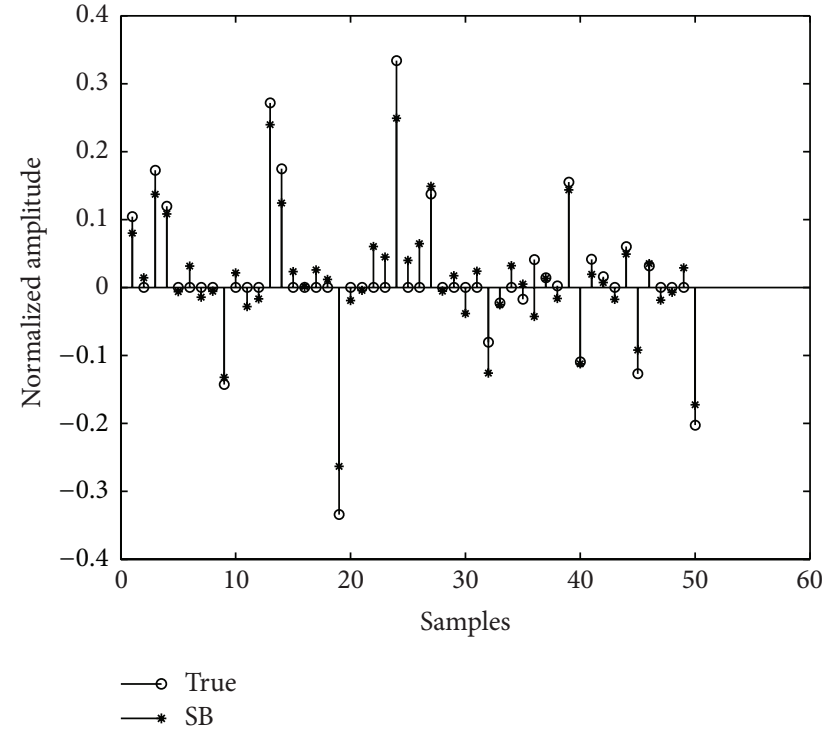

(b)

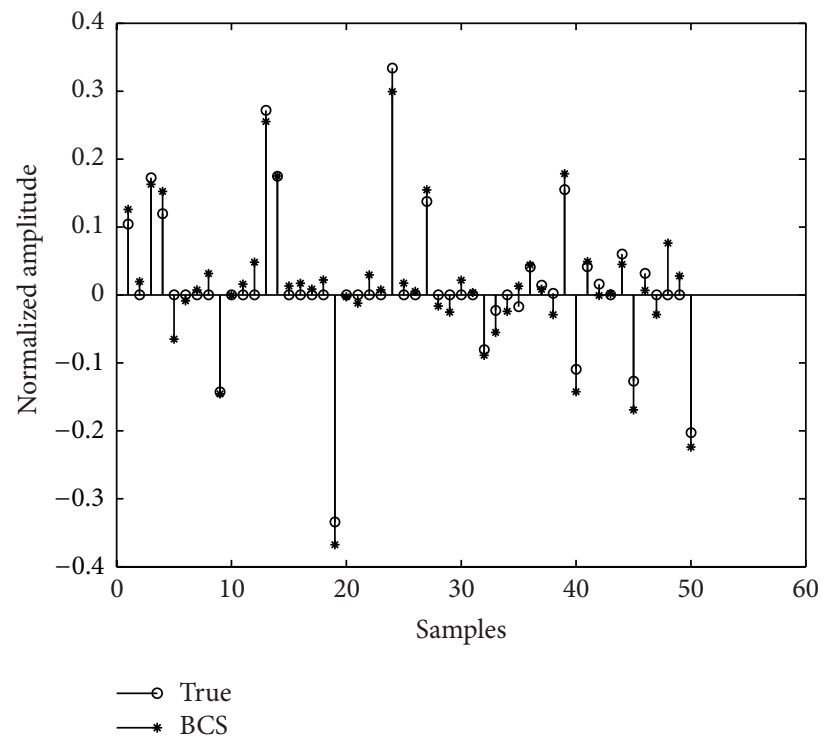

(c)

FIGURE 4: (a) True reflectivity and estimated reflectivity by Bayesian compressive sensing (BCS) (SNR = $10 \mathrm{~dB}$ ). (b) True reflectivity and estimated reflectivity by ML $(\mathrm{SNR}=10 \mathrm{~dB})$. (c) True reflectivity and estimated reflectivity by standard Bayesian $(\mathrm{SNR}=10 \mathrm{~dB})$.

\section{Experiments Results}

In order to demonstrate the performance of the proposed Bayesian compressive sensing algorithm, we give experimental results on both simulated and field data experiments in this section.

5.1. Simulation Data. The proposed Bayesian compressive sensing algorithm can improve the estimations quality compared to ML method and standard Bayesian method in the situation where there are noise and incomplete data. In this section, we support these theoretical arguments with one dimensional synthetic signals simulation experiments.
The proposed Bayesian compressive sensing algorithm is terminated when the criterion $\left\|L\left(\gamma^{k}\right)-L\left(\gamma^{k+1}\right)\right\|^{2}<10^{-4}$ is satisfied. The estimation quality of the three algorithms is compared in the following three parts: the reflectivity sequence recovery, the parameters estimation, and performance indices nMSE. Let us define the signal-to-noise ratios (SNR) as

$$
\mathrm{SNR}=10 \log \left(\frac{\lambda \alpha_{r}^{-1} E(s)}{\alpha_{w}^{-1}}\right)
$$

where $E(s)$ is the seismic wavelet energy and $N$ is the seismic wavelet length, $E(s)=N^{-1}\left(\sum_{i=1}^{N} s_{i}^{2}\right)$. 


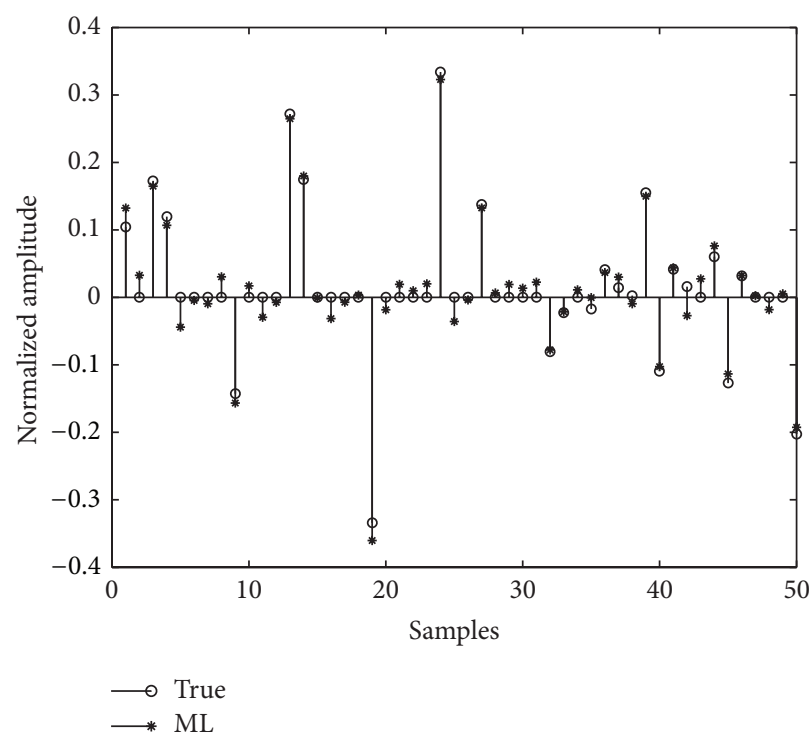

(a)

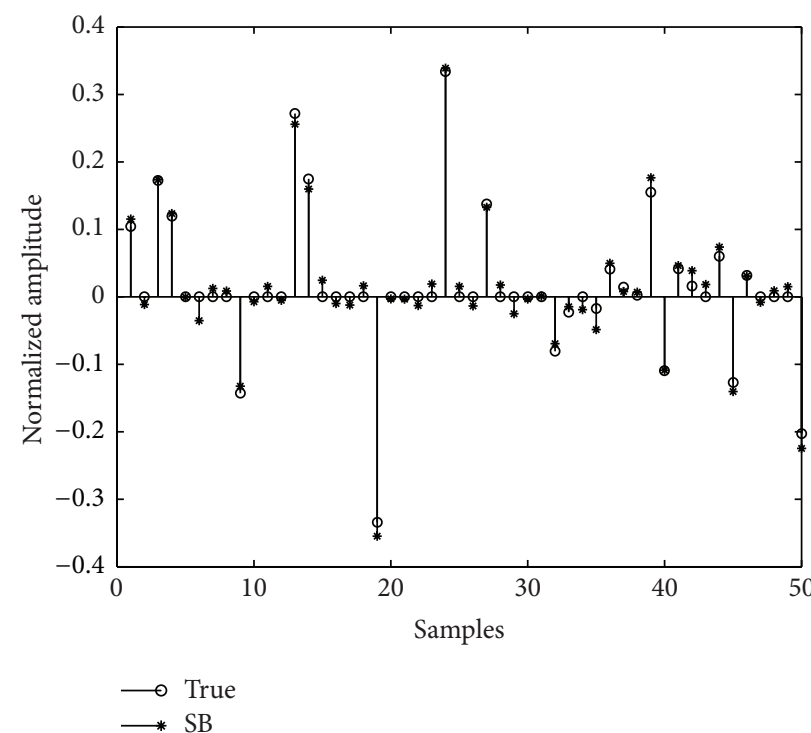

(b)

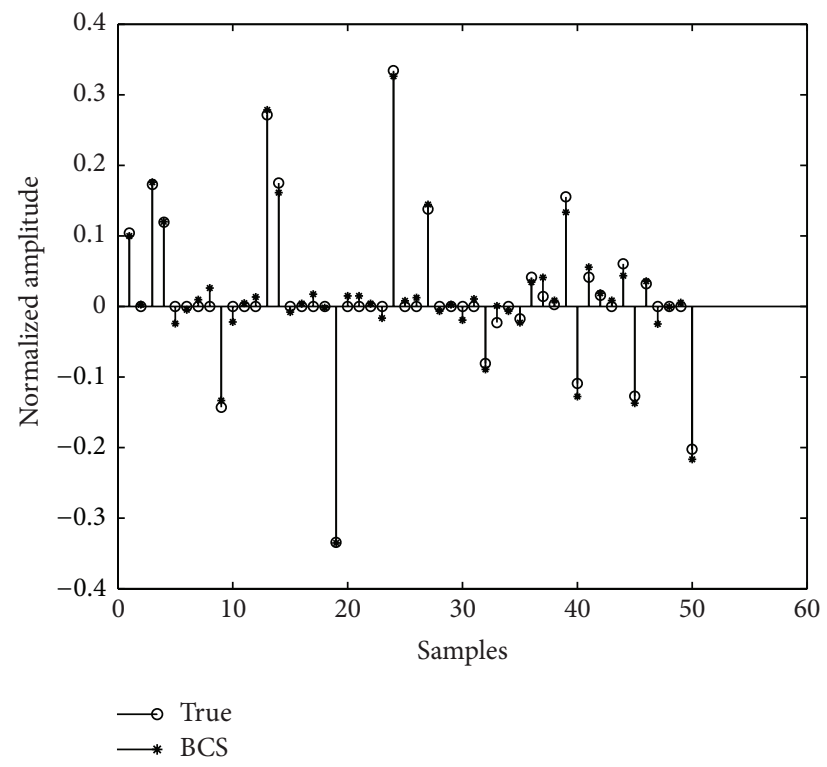

(c)

Figure 5: (a) True reflectivity and estimated reflectivity by Bayesian compressive sensing ( $\mathrm{SNR}=20 \mathrm{~dB}$ ). (b) True reflectivity and estimated reflectivity by $\mathrm{ML}(\mathrm{SNR}=20 \mathrm{~dB})$. (c) True reflectivity and estimated reflectivity by standard Bayesian $(\mathrm{SNR}=20 \mathrm{~dB})$.

A reflectivity sequence with 100 samples by BG model is selected randomly as shown in Figures 2(a) and 3(a). The Stochastic wavelet with 45 samples is shown in Figure 2(b) while the minimum phase wavelet with 35 samples is shown in Figure 3(b). Then, the reflectivity sequence in every figure can be convolved with the corresponding Ricker and minimum phase wavelet and added white Gaussian noise, with SNRs of $10 \mathrm{~dB}$ and $20 \mathrm{~dB}$, respectively. The corresponding seismic records with the noise are shown in Figures 2(c) and $3(\mathrm{c})$, respectively. The reflectivity sequence estimated with $10 \mathrm{~dB}$ and $20 \mathrm{~dB}$ SNRs by the proposed Bayesian compressive sensing algorithm is shown in Figures 2(d) and 3(d), respectively. It can be seen that the Bayesian compressive sensing performs well in reflectivity deconvolution no matter the seismic wavelet is wavelet or minimum phase wavelet.

Figures 4, 5, and 6 show the comparison between the true reflectivity sequence and deconvolved reflectivity sequence by proposed Bayesian compressive sensing method, ML method, and standard Bayesian method with SNRs of $10 \mathrm{~dB}$, $20 \mathrm{~dB}$, and $40 \mathrm{~dB}$, respectively. It can be seen in Figures 4-6 that the Bayesian compressive sensing gives a better reflectivity recovery than the other algorithms. The reflectivity sequence by the Bayesian compressive sensing is more accurate because it has less missed and false detection. When SNR increases, the reflectivity impulse miss number decreases, because both high-amplitude impulse and low-amplitude 


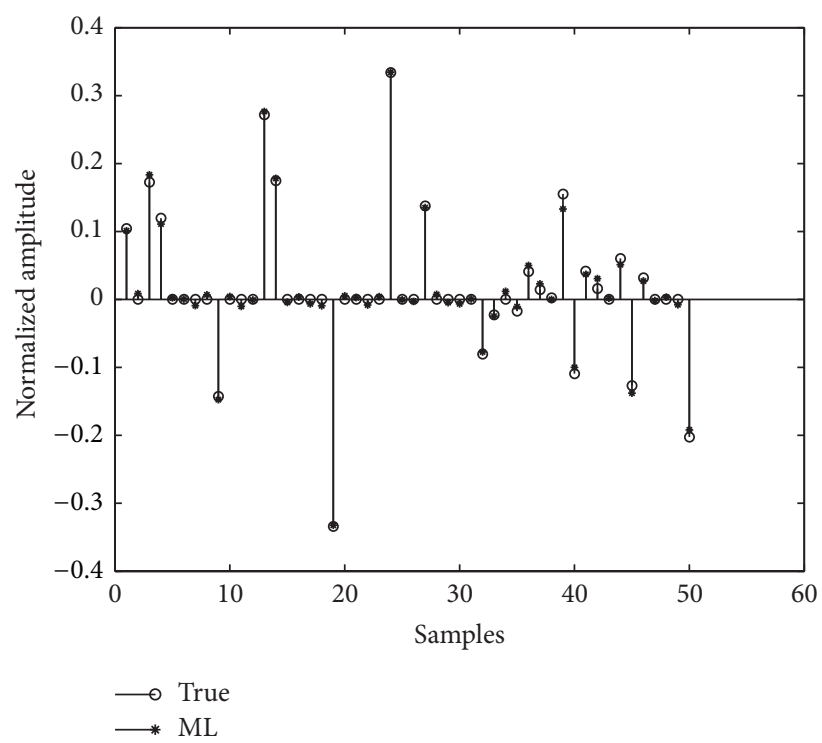

(a)

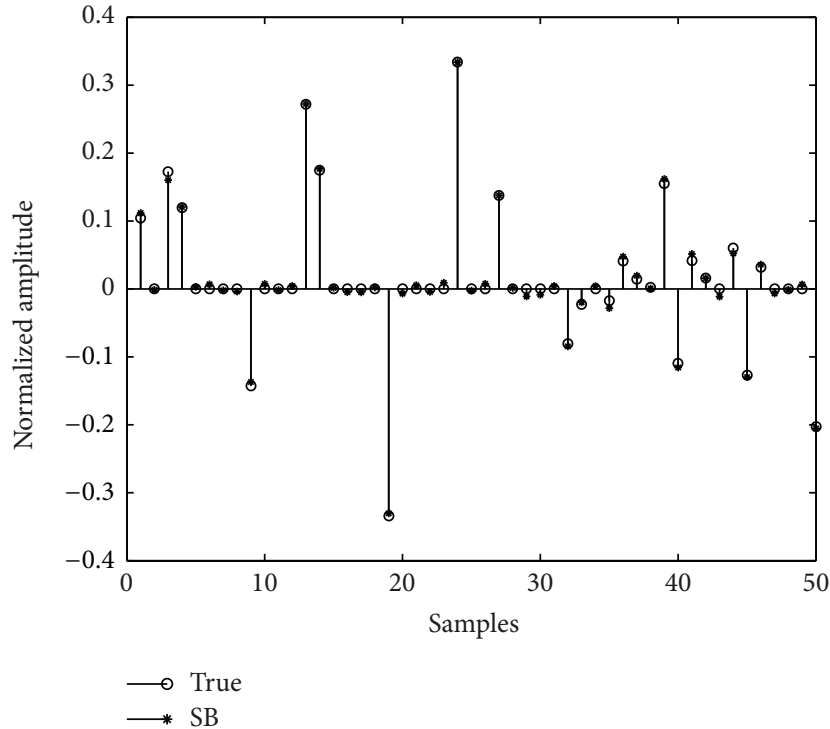

(b)

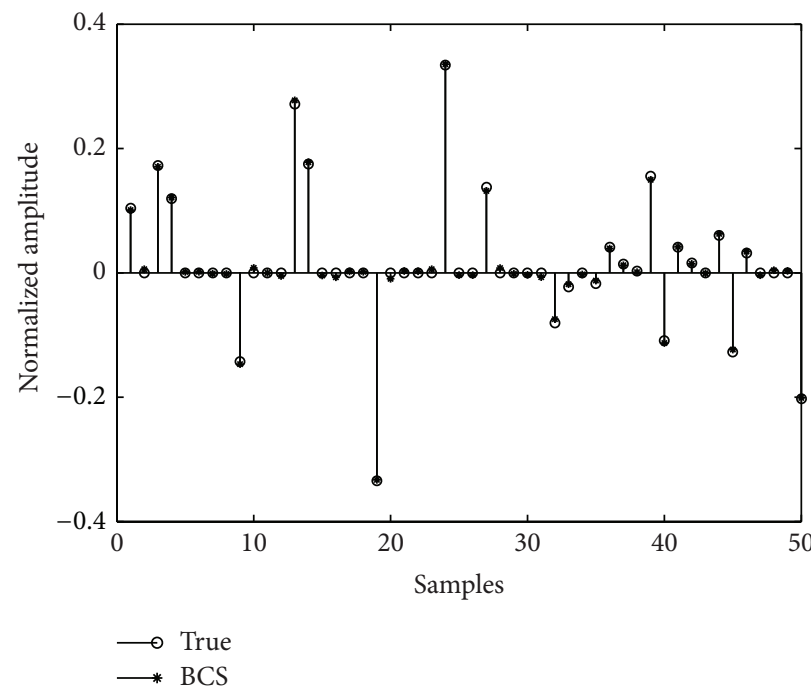

(c)

Figure 6: (a) True reflectivity and estimated reflectivity by Bayesian compressive sensing ( $\mathrm{SNR}=40 \mathrm{~dB}$ ). (b) True reflectivity and estimated reflectivity by $\mathrm{ML}(\mathrm{SNR}=40 \mathrm{~dB})$. (c) True reflectivity and estimated reflectivity by standard Bayesian $(\mathrm{SNR}=40 \mathrm{~dB})$.

impulses are well detected and localized. It can be remarked that the tree algorithms show good robustness with regard to the SNR decrease.

Furthermore, to study improvements brought by the proposed Bayesian compressive sensing algorithm, we consider the following performance indices:

$$
\begin{aligned}
\mathrm{nMSE}_{1} & =\frac{\|r-\widehat{r}\|^{2}}{\|r\|^{2}}, \\
\mathrm{nMSE}_{2} & =\frac{\|r * s-\widehat{r} * \widehat{s}\|^{2}}{\|r * s\|^{2}},
\end{aligned}
$$

where $\mathrm{nMSE}_{1}$ represents the normalized error energy of the estimated wavelet, while $\mathrm{nMSE}_{2}$ represents the normalized error of noiseless data reconstruction. Comparison in
$\mathrm{nMSE}_{1}$ and $\mathrm{nMSE}_{2}$ of the three deconvolution algorithms is shown in Figure 7. The results show the improvement of the performance indices with the Bayesian compressive sensing algorithm compared with the standard Bayesian algorithm and ML algorithm. Although all algorithms show larger errors while SNR decreased, the proposed Bayesian compressive sensing algorithm suffers from noise too, and it is still more reliable than the other algorithms. Moreover, the Bayesian compressive sensing behaves much better when there is almost no noise, because the Bayesian compressive sensing uses the signals prior knowledge and the linear wavelet transform.

5.2. Field Data Experiments. Figure 8(a) shows a real CDP stack seismic profile. The reflectivity estimates obtained by 


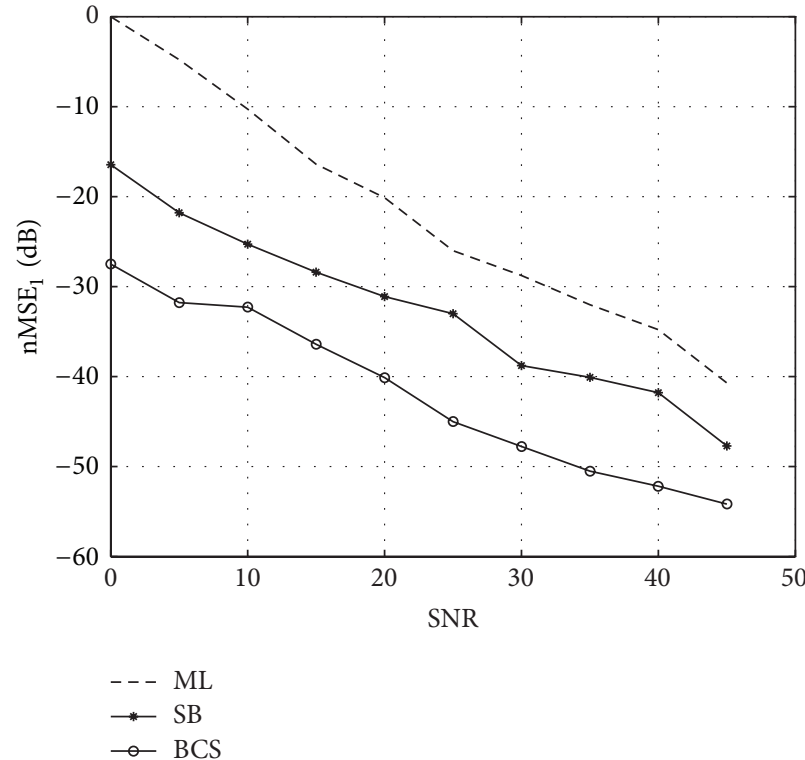

(a)

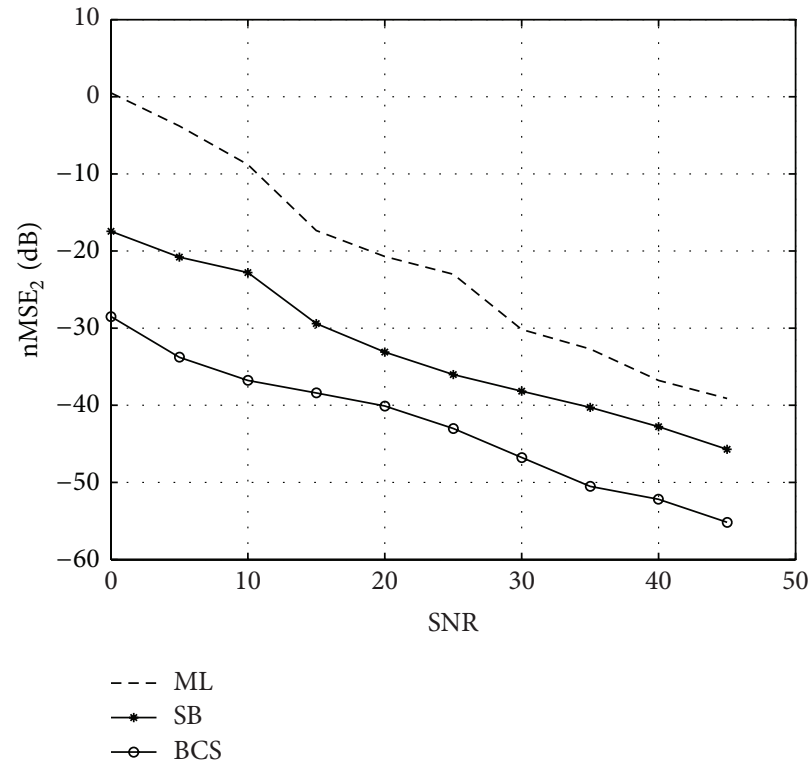

(b)

FIGURE 7: $\mathrm{nMSE}_{1}$ and $\mathrm{nMSE}_{2}$ performance indices for $\mathrm{ML}$ algorithm, standard Bayesian, and Bayesian compressive sensing.

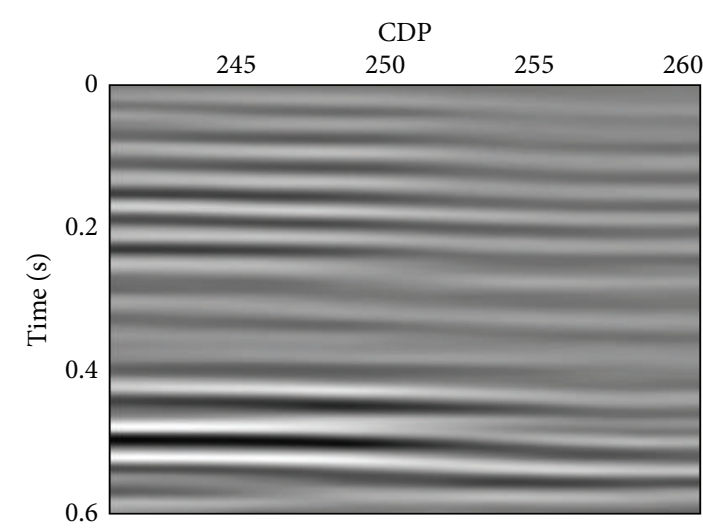

(a)

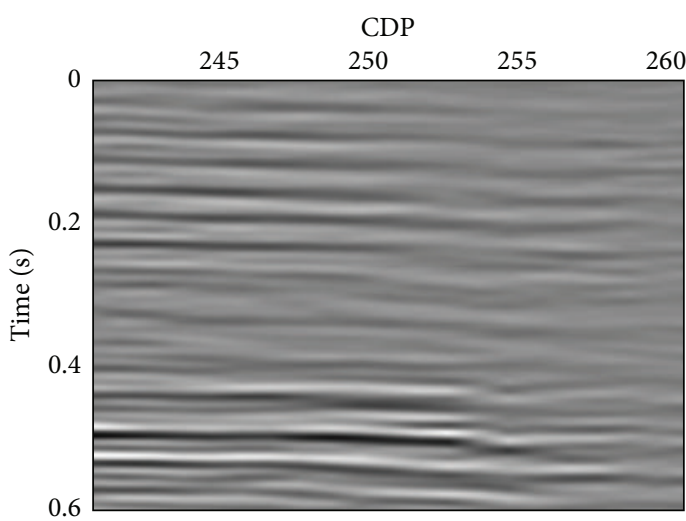

(c)

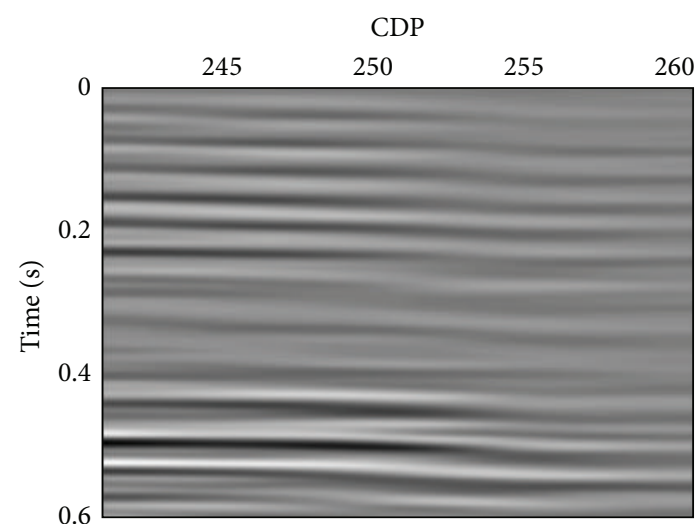

(b)

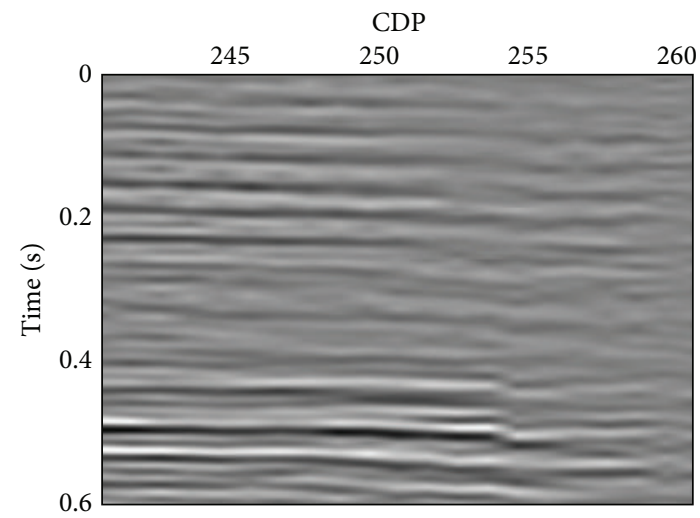

(d)

Figure 8: (a) A real CDP profile. (b) Reflectivity estimates obtained by ML algorithm. (c) Reflectivity estimates obtained by variational Bayesian algorithm. (d) Reflectivity estimates obtained by Bayesian compressive sensing algorithm. 
ML algorithm, variational Bayesian, and Bayesian compressive sensing are shown in Figures 8(b), 8(c), and 8(d), respectively. Because the true layer structure is unknown, the continuous nature of the channel estimates obtained by the proposed algorithm can be seen. New layers become much clearer in the deconvoluted seismic image by variational Bayesian algorithm. The translations between layers are much thinner and less blurred in the deconvoluted seismic image by Bayesian compressive sensing algorithm, especially near CDP 225 at $0.5 \mathrm{~s}$.

\section{Conclusions}

Convolution and additive Gaussian noises are the major problems in blind seismic signal processing. In order to solve two problems, a novel blind seismic deconvolution algorithm based on Bayesian compressive sensing has been proposed in this paper. Bayesian modeling and the optimization method are used to estimate the unknown reflectivity sequence, seismic wavelet, and hyperparameters simultaneously in the proposed algorithm. Furthermore, we compared the proposed Bayesian compressive sensing algorithm with ML algorithm and standard Bayesian algorithm. Seismic images are much clear and accurate by the proposed Bayesian compressive sensing algorithm on both simulation data and field data.

\section{Conflict of Interests}

The authors declare that there is no conflict of interests regarding the publication of this paper.

\section{References}

[1] J. Ma, "Compressed sensing for surface characterization and metrology," IEEE Transactions on Instrumentation and Measurement, vol. 59, no. 6, pp. 1600-1615, 2010.

[2] M. F. Duarte and R. G. Baraniuk, "Spectral compressive sensing," Applied and Computational Harmonic Analysis, vol. 35, no. 1, pp. 111-129, 2013.

[3] Y. Erlich, A. Gordon, M. Brand, G. J. Hannon, and P. P. Mitra, "Compressed genotyping," IEEE Transactions on Information Theory, vol. 56, no. 2, pp. 706-723, 2010.

[4] A. Amir and O. Zuk, "Bacterial community reconstruction using compressed sensing," Journal of Computational Biology, vol. 18, no. 11, pp. 1723-1741, 2011.

[5] M. F. Duarte, M. A. Davenport, D. Takbar et al., "Single-pixel imaging via compressive sampling: building simpler, smaller, and less-expensive digital cameras," IEEE Signal Processing Magazine, vol. 25, no. 2, pp. 83-91, 2008.

[6] M. A. Herman and T. Strohmer, "High-resolution radar via compressed sensing," IEEE Transactions on Signal Processing, vol. 57, no. 6, pp. 2275-2284, 2009.

[7] L. McMackin, M. A. Herman, B. Chatterjee, and M. Weldon, "A high-resolution SWIR camera via compressed sensing," in Infrared Technology and Applications XXXVIII, Proceedings of SPIE, pp. 835303-835303-10, Baltimore, Md, USA, 2012.

[8] E. J. Candès, J. Romberg, and T. Tao, "Robust uncertainty principles: exact signal reconstruction from highly incomplete frequency information," IEEE Transactions on Information Theory, vol. 52, no. 2, pp. 489-509, 2006.
[9] D. L. Donoho, "Compressed sensing," IEEE Transactions on Information Theory, vol. 52, no. 4, pp. 1289-1306, 2006.

[10] W. Qisong, Y. D. Zhang, M. G. Amin, and B. Himed, "Multitask bayesian compressive sensing exploiting intra-task dependency," IEEE Signal Processing Letters, vol. 22, no. 4, pp. 430434, 2015.

[11] S. D. Babacan, R. Molina, and A. K. Katsaggelos, "Bayesian compressive sensing using Laplace priors," IEEE Transactions on Image Processing, vol. 19, no. 1, pp. 53-63, 2010.

[12] S. Minato, T. Matsuoka, and T. Tsuji, "Singular-value decomposition analysis of source illumination in seismic interferometry by multidimensional deconvolution," Geophysics, vol. 78, no. 3, pp. Q25-Q34, 2013.

[13] S. H. Ju, "A deconvolution scheme for determination of seismic loads in finite-element analyses," Bulletin of the Seismological Society of America, vol. 103, no. 1, pp. 258-267, 2013.

[14] G. M. Menanno and A. Mazzotti, "Deconvolution of multicomponent seismic data by means of quaternions: theory and preliminary results," Geophysical Prospecting, vol. 60, no. 2, pp. 217-238, 2012.

[15] K. Wapenaar, J. van der Neut, E. Ruigrok et al., "Seismic interferometry by crosscorrelation and by multidimensional deconvolution: a systematic comparison," Geophysical Journal International, vol. 185, no. 3, pp. 1335-1364, 2011.

[16] B. Nsiri, T. Chonavel, J.-M. Boucher, and H. Nouzé, "Blind submarine seismic deconvolution for long source wavelets," IEEE Journal of Oceanic Engineering, vol. 32, no. 3, pp. 729-743, 2007.

[17] Y. Li and G. Zhang, "Blind seismic deconvolution using variational Bayesian method," Journal of Applied Geophysics, vol. 110, pp. 82-89, 2014.

[18] S. Yildirim, A. T. Cemgil, M. Aktar, Y. Özakin, and A. Ertüzün, "A Bayesian deconvolution approach for receiver function analysis," IEEE Transactions on Geoscience and Remote Sensing, vol. 48, no. 12, pp. 4151-4163, 2010.

[19] S. Yuan and S. Wang, "Spectral sparse Bayesian learning reflectivity inversion," Geophysical Prospecting, vol. 61, no. 4, pp. 735-746, 2013.

[20] E. J. Candès and M. B. Wakin, "An introduction to compressive sampling," IEEE Signal Processing Magazine, vol. 25, no. 2, pp. 21-30, 2008.

[21] C. P. Robert, The Bayesian Choice, Springer, New York, NY, USA, 2007.

[22] A. Gholami, "Residual statics estimation by sparsity maximization," Geophysics, vol. 78, no. 1, pp. V11-V19, 2013.

[23] D. L. Wang, Z. F. Tong, C. Tang, and H. Zhu, "An iterative curvelet thresholding algorithm for seismic random noise attenuation," Applied Geophysics, vol. 7, no. 4, pp. 315-324, 2010.

[24] A. Heimer and I. Cohen, "Multichannel seismic deconvolution using Markov-Bernoulli random-field modeling," IEEE Transactions on Geoscience and Remote Sensing, vol. 47, no. 7, pp. 2047-2058, 2009.

[25] A. T. Cemgil, C. Févotte, and S. J. Godsill, "Variational and stochastic inference for Bayesian source separation," Digital Signal Processing, vol. 17, no. 5, pp. 891-913, 2007.

[26] S. Kim and C. D. Yoo, "Underdetermined blind source separation based on subspace representation," IEEE Transactions on Signal Processing, vol. 57, no. 7, pp. 2604-2614, 2009.

[27] C. Févotte and S. J. Godsill, "A Bayesian approach for blind separation of sparse sources," IEEE Transactions on Audio, Speech and Language Processing, vol. 14, no. 6, pp. 2174-2188, 2006. 
[28] C. Févotte, Bayesian Audio Source Separation, Springer, New York, NY, USA, 2007.

[29] D. Tzikas, A. Likas, and N. Galatsanos, "Variational bayesian blind image deconvolution with student-t priors," in Proceedings of the 14th IEEE International Conference on Image Processing (ICIP '07), pp. I109-I112, IEEE, San Antonio, Tex, USA, September 2007.

[30] M. Beal, Variational algorithms for approximate Bayesian inference [Ph.D. thesis], Gatsby Computational Neuroscience Unit, Univercity College, London, UK, 2003.

[31] B. Amizic, L. Spinoulas, R. Molina, and A. K. Katsaggelos, "Compressive blind image deconvolution," IEEE Transactions on Image Processing, vol. 22, no. 10, pp. 3994-4006, 2013.

[32] O. Rosec, J.-M. Boucher, B. Nsiri, and T. Chonavel, "Blind marine seismic deconvolution using statistical MCMC methods," IEEE Journal of Oceanic Engineering, vol. 28, no. 3, pp. 502512, 2003.

[33] N. Kazemi and M. D. Sacchi, "Sparse multichannel blind deconlution," Geophysics, vol. 79, no. 5, pp. 143-152, 2014. 


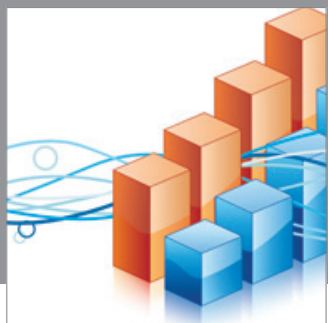

Advances in

Operations Research

mansans

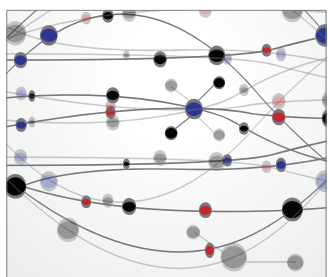

The Scientific World Journal
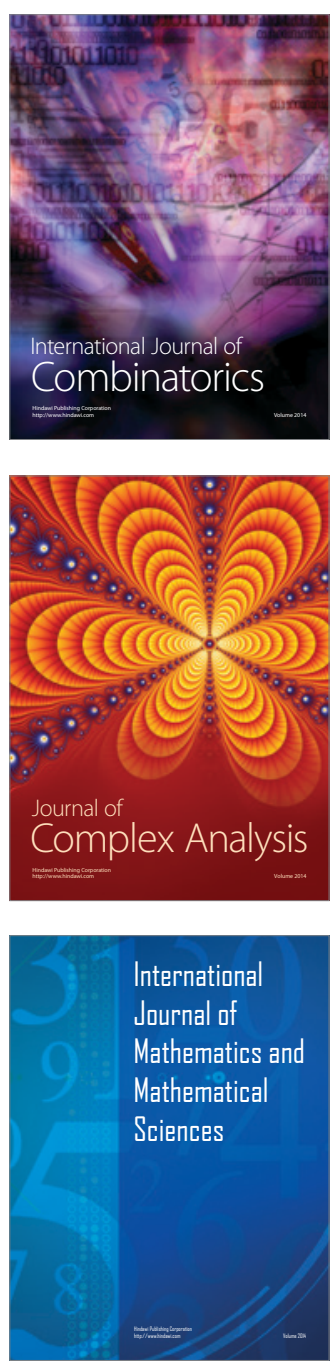
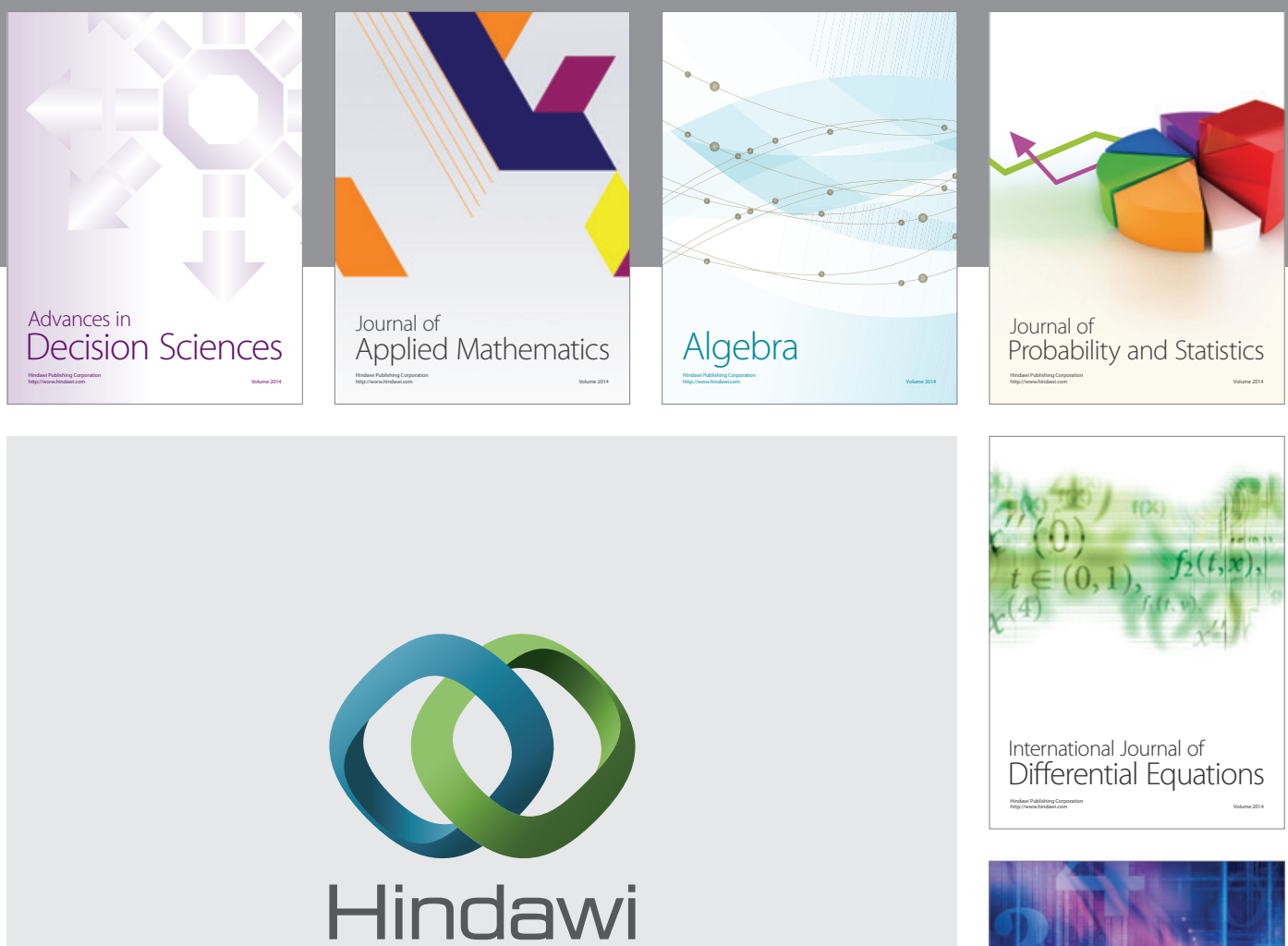

Submit your manuscripts at http://www.hindawi.com
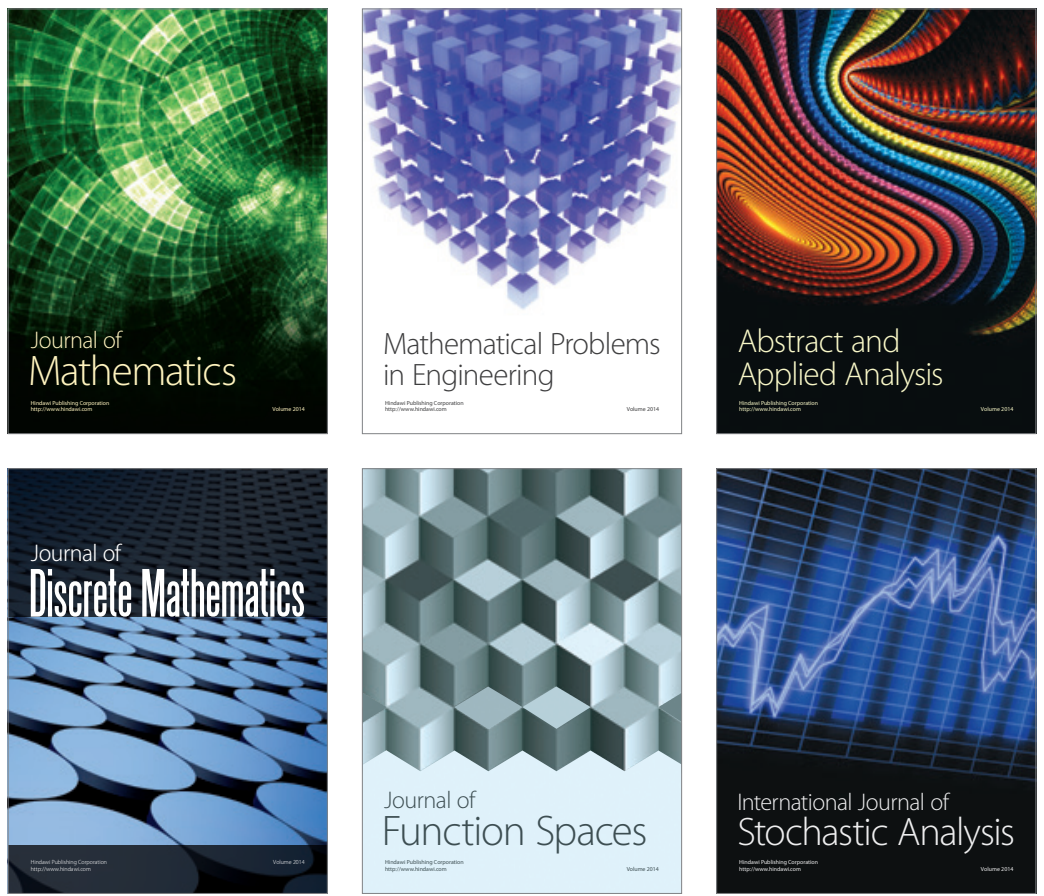

Journal of

Function Spaces

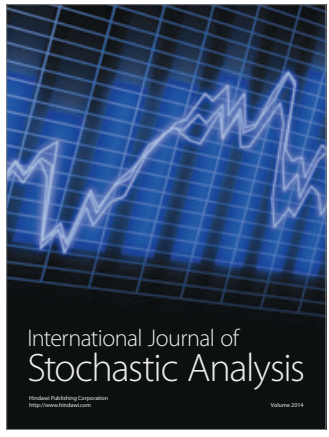

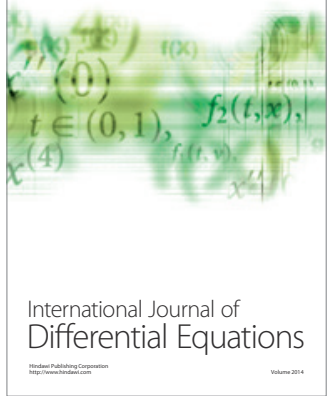
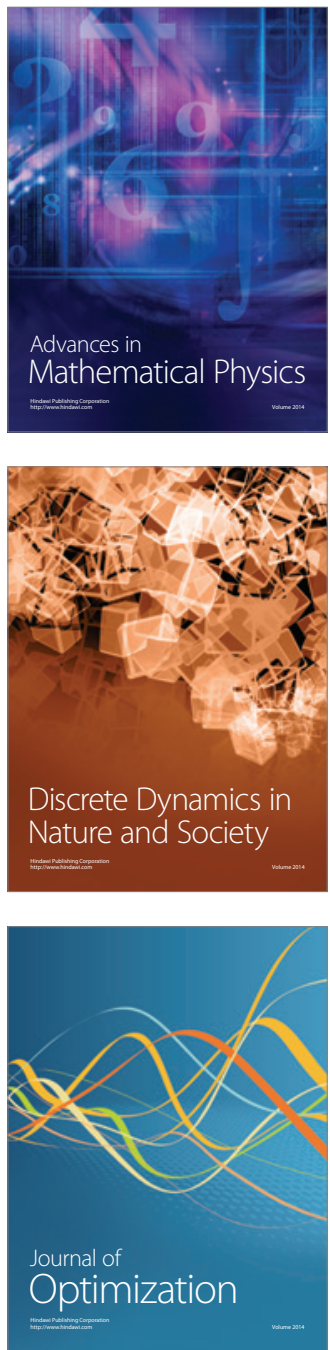